\title{
Microhabitat branchial préférentiel de Microcotyle chrysopbrii
}

\author{
van Beneden et Hesse, 1863
}

(Monogenea, Microcotylidae)

parasite de la Daurade (Sparus aurata L., 1758)

\author{
par D. NOISY (*) et Cl. MAILLARD (**) \\ * Institut de Biologie animale, Université de Lausanne, 1005 Lausanne (Suisse). \\ ** Laboratoire de Parasitologie comparée, \\ Université des Sciences et Techniques du Languedoc, \\ place E. Bataillon, 34060 Montpellier Cedex (France).
}

\section{Résumé.}

L'analyse des infestations naturelles d'une population de Daurades capturées dans l'Etang de Thau (Sète) a été effectuée. Elle montre qu'on peut distinguer deux catégories d'hôtes selon le type d'infestation qui les affecte. Chez les Poissons de petite taille, Microcotyle chrysophrii se fixe préférentiellement sur les arcs branchiaux I et IV alors que chez les hôtes de grande taille, le parasite régresse de l'arc IV. Ces différences de répartition branchiale sont probablement liées à la maturité sexuelle de Sparus aurata.

\section{Summary.}

The preferential gill microhabitat of Microcotyle chrysophrii van Beneden and Hesse, 1863 (Monogenea, Microcotylidae) parasite of the gilthead (Sparus aurata L., 1758).

Natural infestations of a population of the Teleost Sparus aurata caught from Etang de Thau (Sète) were analysed. Two categories of host are distinguishable according to the type of infestation. On short length fishes, Microcotyle chrysophrii is rather hooked on the first and the last gill whereas on long lenght fishes the parasite diminishes from the last gill. These two different gill distributions are likely related to the sexual maturity of Sparus aurata.

Accepté le 15 octobre 1979.

Annales de Parasitologie humaine et comparée (Paris), t. 55, $\mathrm{n}^{\circ} 1$. 
M. chrysophrii est un Monogène appartenant à la famille des Microcotylidae. La description originale de ce parasite branchial a été faite par Van Beneden et Hesse en 1863. Euzet et Noisy (sous presse) ont apporté des précisions sur l'anatomie de cet animal.

\section{Matériel et méthodes}

Le parasite se récolte fréquemment chez 4 Sparidae pêchés dans l'Etang de Thau (Sète) : Diplodus sargus, D. annularis, D. vulgaris et Sparus aurata. Cependant, si chez les trois Diplodus, $M$. chrysophrii coexiste avec d'autres Microcotylidae des genres Atriaster et Atrispinum, l'infestation monospécifique ne s'observe que chez la Daurade (S. aurata).

Nous rapportons dans ce travail les résultats des infestations naturelles monospécifiques.

La méthode suivie est identique à celle qui a été utilisée par Lambert et Maillard (1975) dans l'étude du microhabitat de deux Monopisthocotylea parasites de Dicentrarchus labrax. Nous avons cependant modifié le découpage par secteur de l'arc branchial en utilisant non pas 5 mais 3 secteurs numérotés de 1 à 3 : secteurs dorsal, médian et ventral. Les auteurs précédents avaient adopté un découpage en 5 secteurs mieux approprié à l'étude des Monogènes Monopisthocotylea plus petits et surtout plus nombreux.

D'autre part, nous abandonnons la répartition par zone, car le hapteur de l'adulte est si développé qu'il rend impossible la localisation par zone de l'animal sur le filament branchial.

Un test de signification $\left(\chi^{2}\right)$ a été appliqué aux répartitions observées. Il est effectué à partir d'un tableau de contingence et en utilisant la table de Fisher et Yates.

\section{Résultats}

Nous avons pu examiner 121 Daurades provenant de l'Etang de Thau. 103 d'entre elles étaient parasitées, soit une prévalence globale de $85 \%$. La quasi totalité de ces Poissons a été disséquée pendant les mois d'octobre et novembre 1976 et 1977, c'est-à-dire au cours de leur migration automnale vers la mer.

1090 Microcotyle chrysophrii ont été récoltés, soit une abondance globale de 10,58 .

Une analyse plus détaillée de ces premiers résultats montre cependant qu'il existe une différence significative dans la répartition et l'abondance des parasites selon la taille des hôtes. Nous avons déterminé que le microhabitat préférentiel de M. chrysophrii diffère lorsque les Daurades atteignent la taille de $25 \mathrm{~cm}$. 
Nous examinerons donc la localisation du parasite dans la cavité branchiale des $S$. aurata de taille inférieure à $25 \mathrm{~cm}$ ( 85 individus) et de taille supérieure ou égale à $25 \mathrm{~cm}$ (36 individus).

\section{Répartition chez les Daurades inférieures à $25 \mathrm{~cm}$.}

Parmi les 85 Daurades examinées, dont la longueur varie de 10 à $24 \mathrm{~cm}, 72$ d'entre elles étaient porteuses de Microcotylidae, soit une prévalence de $85 \%$.

891 individus de ce parasite ont été récoltés, soit une abondance égale à 12,38.

Les répartitions sont rapportées dans les tableaux suivants :

- Répartition par arc (fig. 1).

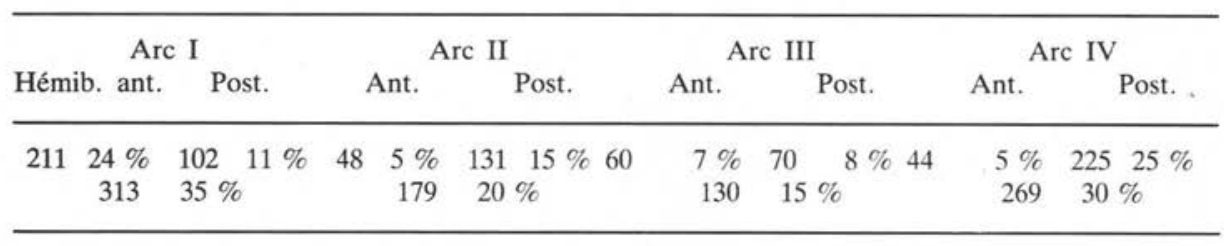
ficative.

Les côtés droit et gauche sont groupés, car il n'existe pas de différence signi-

- Répartition par secteur (fig. 2, 3, 4, 5).

\begin{tabular}{rrrrrrrrr}
\hline & \multicolumn{2}{c}{ Arc I } & \multicolumn{2}{c}{ Arc II } & \multicolumn{2}{c}{ Arc III } & \multicolumn{2}{c}{ Arc IV } \\
\hline $\mathrm{S}_{1}$ & 128 & $41 \%$ & 96 & $54 \%$ & 82 & $63 \%$ & 197 & $73 \%$ \\
\hline $\mathrm{S}_{2}$ & 97 & $31 \%$ & 54 & $30 \%$ & 29 & $22 \%$ & 25 & $9 \%$ \\
\hline $\mathrm{S}_{3}$ & 88 & $28 \%$ & 29 & $16 \%$ & 19 & $15 \%$ & 47 & $17 \%$ \\
\hline
\end{tabular}

Ainsi, $M$. chrysophrii se fixe préférentiellement sur l'hémibranchie antérieure du premier arc $(24 \%)$ et l'hémibranchie postérieure du quatrième arc $(25 \%)$. De plus, quel que soit l'arc branchial considéré, c'est le secteur dorsal qui présente la plus grande densité de parasite.

La répartition antéro-postérieure et dorso-ventrale de ce Monogène est donc nettement définie.

\section{Répartition chez les Daurades supérieures à $25 \mathrm{~cm}$.}

Nous avons disséqué 36 Daurades dont la taille est comprise entre 25 et $47 \mathrm{~cm}$. Dans cet échantillonnage, ce sont les individus de 27 et $28 \mathrm{~cm}$ qui sont les plus nombreux. 

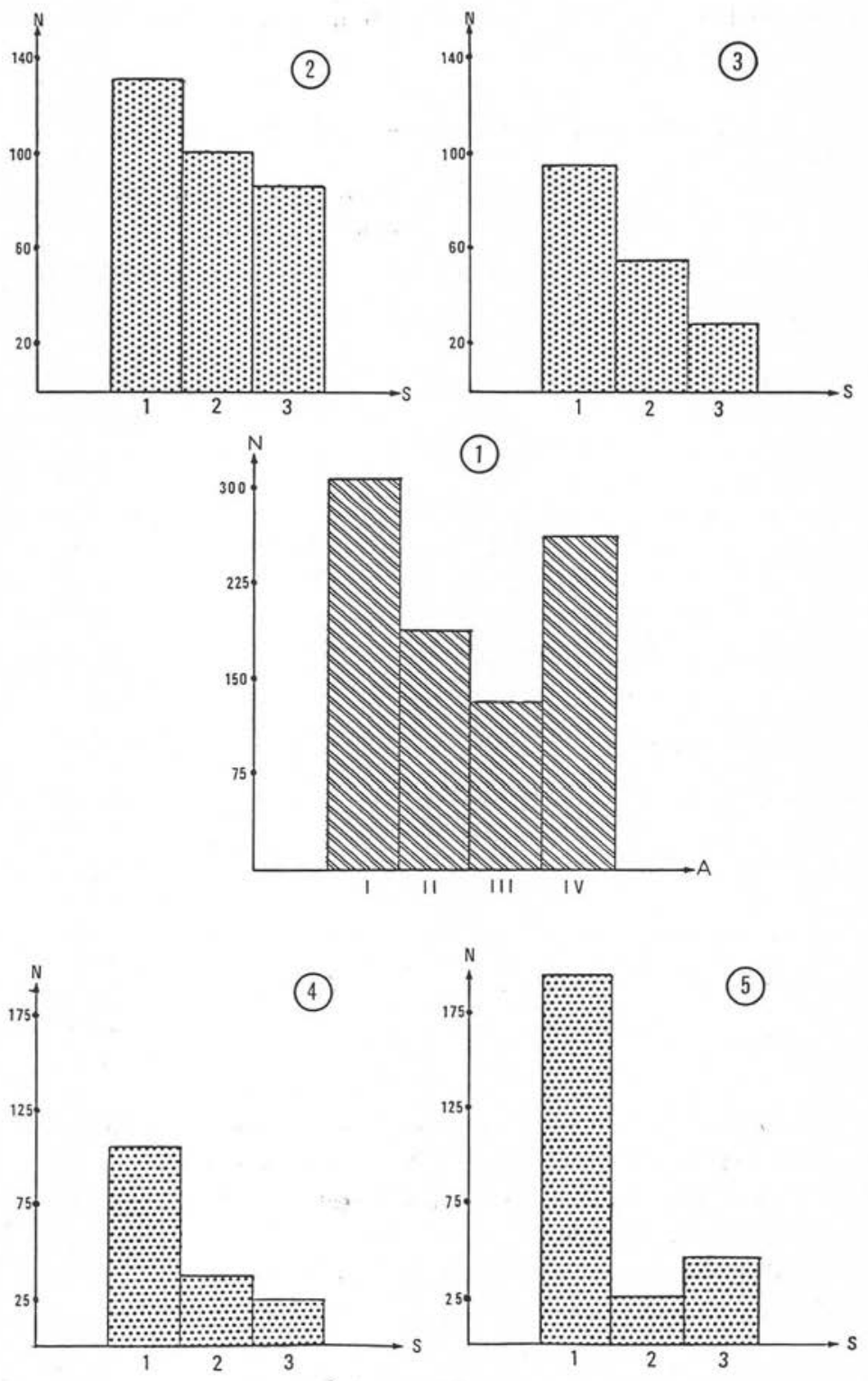

Fig. 1 à 5 : répartition de Microcotyle chrysophrii chez les Sparus aurata de taille supérieure à $25 \mathrm{~cm} ; 1$ : répartition par arc ; 2 : répartition par secteur sur l'arc I ; 3 : répartition par secteur sur l'arc II ; 4 : répartition par secteur sur l'arc III ; 5 : répartition par secteur sur l'arc IV. 

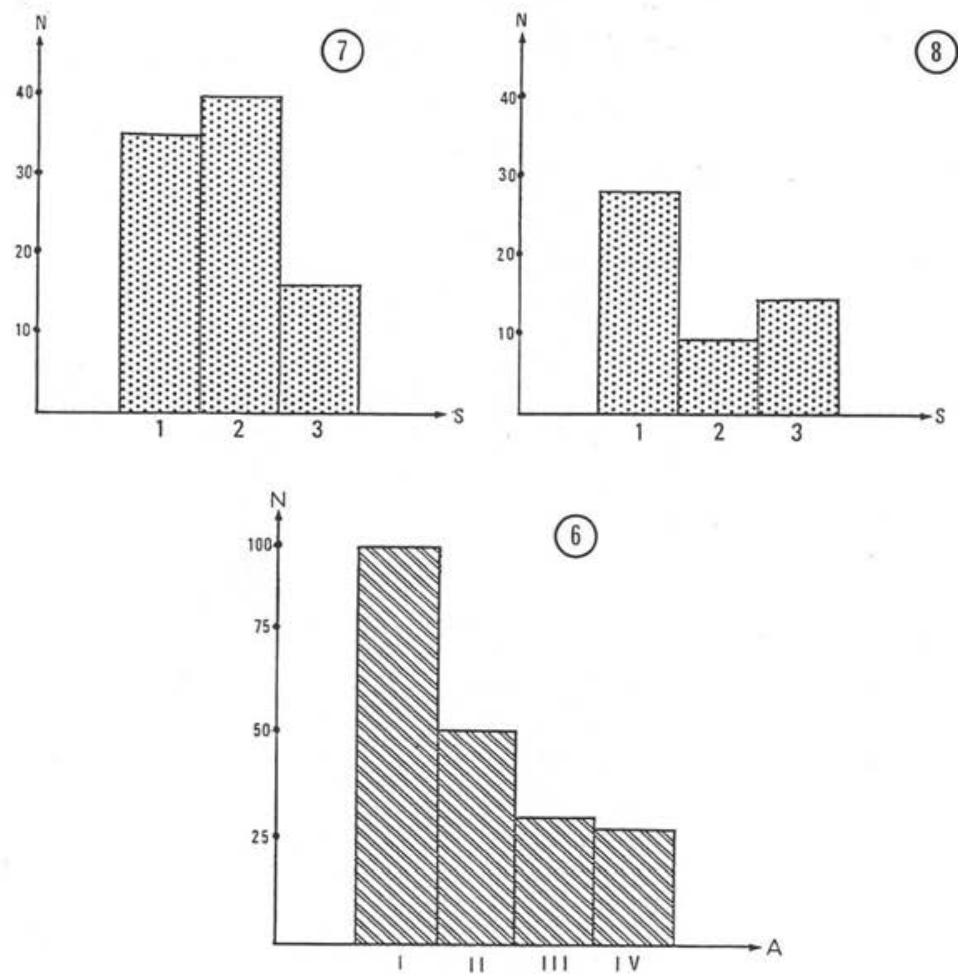

8
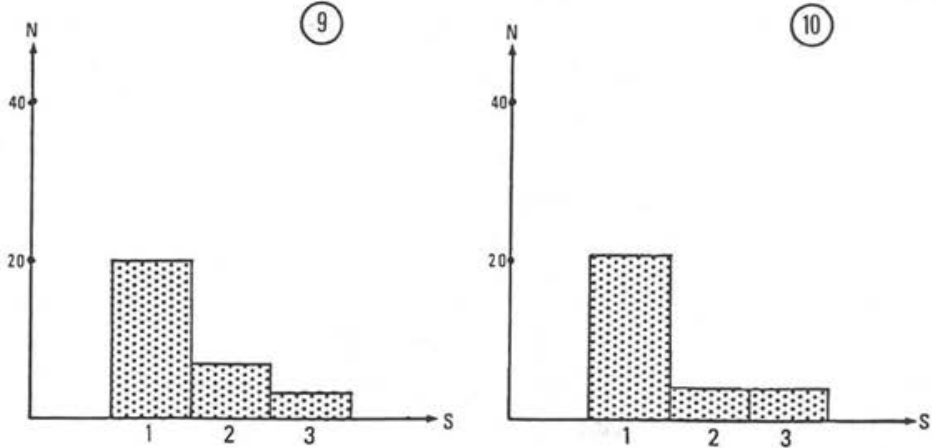

Fig. 6 à 10 : répartition de Microcotyle chrysophrii chez les Sparus aurata de taille inférieure à $25 \mathrm{~cm} ; 6$ : répartition par arc ; $7:$ répartition par secteur sur l'arc I ; 8 : répartition par secteur sur l'arc II ; 9 : répartition par secteur sur l'arc III ; 10 : répartition par secteur sur l'arc IV. 
31 Poissons étaient parasités, soit une prévalence de $86 \% .199$ parasites ont été récoltés, soit une abondance égale à 6,42 .

Les répartitions observées dans ce cas sont les suivantes :

- Répartition par arc (fig. 6).

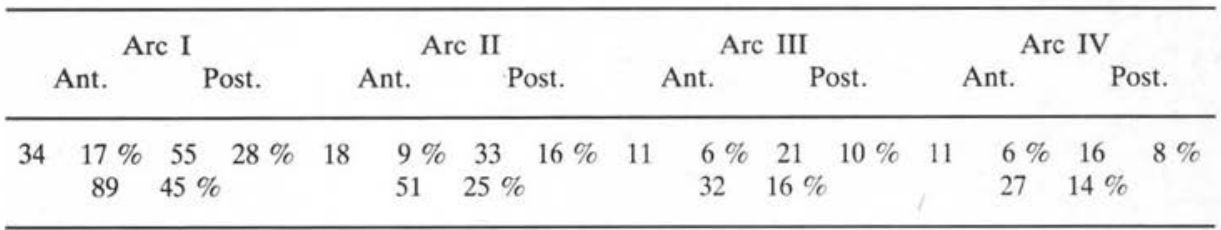

- Répartition par secteur (fig. 7, 8, 9, 10):

\begin{tabular}{rrrrrrrrr}
\hline & \multicolumn{2}{c}{ Arc I } & \multicolumn{2}{c}{ Arc II } & Arc III & \multicolumn{2}{c}{ Arc IV } \\
\hline $\mathrm{S}_{1}$ & 34 & $38 \%$ & 27 & $53 \%$ & 20 & $63 \%$ & 21 & $78 \%$ \\
\hline $\mathrm{S}_{2}$ & 40 & $45 \%$ & 9 & $18 \%$ & 7 & $22 \%$ & 3 & $11 \%$ \\
\hline $\mathrm{S}_{3}$ & 15 & $17 \%$ & 15 & $29 \%$ & 5 & $16 \%$ & 3 & $11 \%$ \\
\hline
\end{tabular}

On peut donc observer que dans ce cas, $M$. chrysophrii se répartit par arc selon un gradient antéro-postérieur décroissant. Le parasite est essentiellement présent sur l'hémibranchie postérieure du premier arc $(28 \%)$.

D'autre part, sur tous les arcs branchiaux hormis le premier, c'est toujours sur le secteur dorsal que le plus grand nombre de Microcotylidae est récolté.

\section{Discussion}

Le test du $\chi^{2}$ appliqué aux deux répartitions par arc indique que celles-ci diffèrent de manière très significative $\left(\chi^{2}=28,64\right.$ pour $\left.\alpha=5 \%\right)$.

Le parasite de la Daurade n'adopte donc pas la même répartition par arc selon qu'il se trouve sur un hôte de moins de $25 \mathrm{~cm}$ ou de plus de $25 \mathrm{~cm}$.

Nous ne pouvons pas pour l'instant proposer une explication à ce phénomène, mais deux hypothèses peuvent être avancées.

D'après les travaux sur la physiologie sexuelle de la Daurade (D'Ancona, 1950, Audouin, 1962), il existe chez ces Poissons un hermaphrodisme protandrique. La première maturation sexuelle (testiculaire) se fait au cours de la seconde année de vie. 
La courbe de répartition de $M$. chrysophrii change d'allure lorsque les hôtes atteignent la taille de $25 \mathrm{~cm}$. Cette taille, d'après Lassère (1974), correspond au début de la maturation des gonades de la Daurade dans l'Etang de Thau (fig. 11). L'apparition des hormones testiculaires dans le sang modifierait le comportement de ce parasite hématophage.

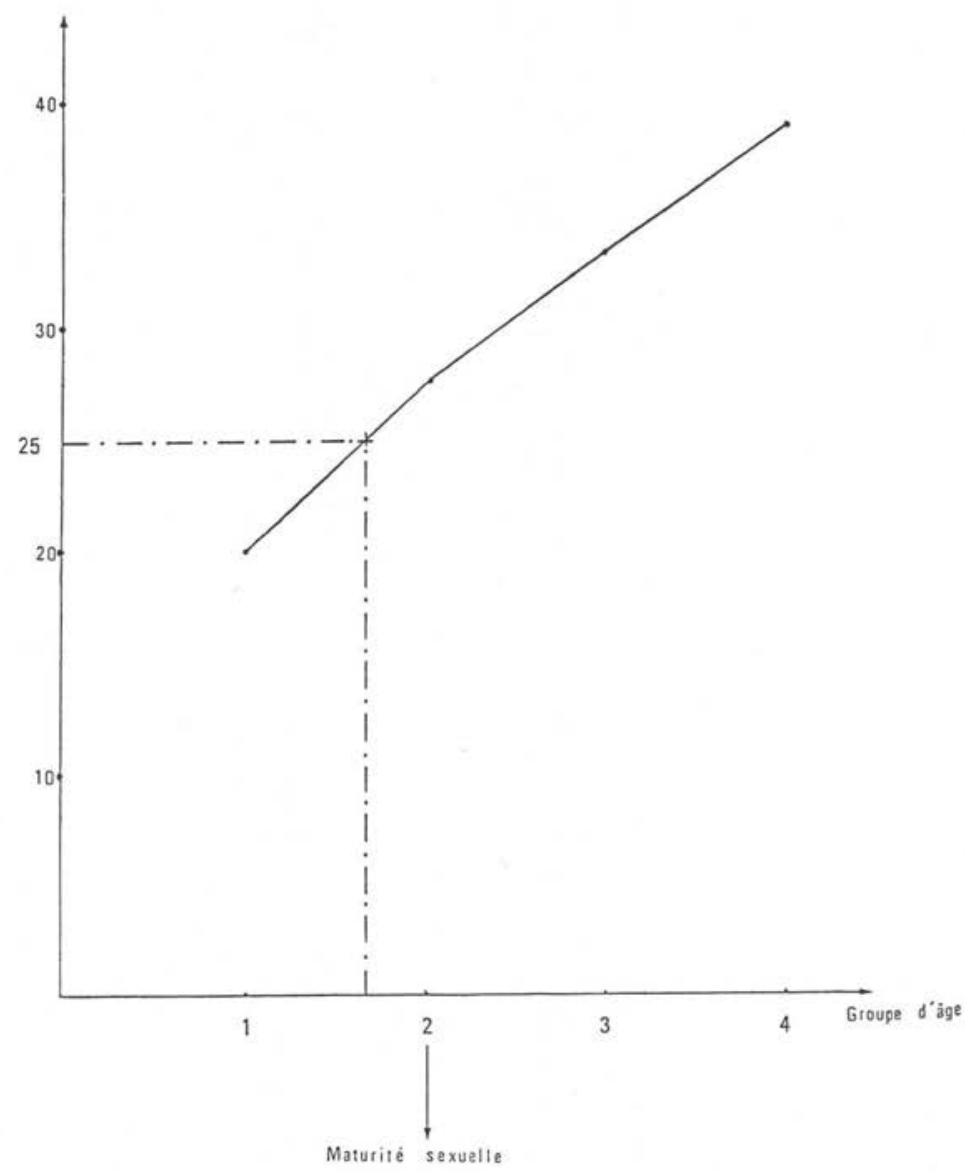

Fig. 11. Croissance en longueur de Sparus aurata dans l'Etang de Thau (d'après Lasserre).

La maturité sexuelle agirait également sur l'abondance du parasite puisque celleci passe de 12,58 (Daurades de moins de $25 \mathrm{~cm}$ ) à 6,42 (hôtes de plus de $25 \mathrm{~cm}$ ). Un tel phénomène n'a été que rarement signalé chez les Monogènes où l'on observe généralement soit une stabilité, soit une augmentation progressive de l'abondance avec la taille. Cependant, Llewellyn (1962) décrit un cas semblable, c'est-à-dire une dimi- 
nution de l'abondance de Gastrocotyle trachuri en relation avec l'augmentation de l'âge de l'hôte, Trachurus trachurus.

On peut aussi envisager l'hypothèse de deux espèces jumelles au sein de l'espèce Microcotyle chrysophrii. Nous serions en présence d'une population dite L (par l'allure de la représentation en histogramme) qui a pour optimum l'arc I et d'une population J inféodée principalement à l'arc IV.

Les formes L et $\mathrm{J}$ seraient présentes simultanément chez les Daurades de petite taille et l'abondance de 12,38 serait la somme des abondances de $M$. chrysophrii $\mathrm{L}$. et J. Chez les Daurades ayant atteint la maturité sexuelle, seule la forme L subsisterait.

Cette seconde hypothèse n'explique cependant pas la régression de la population J chez les Sparus aurata de grande taille.

Toutes les tentatives effectuées pour mettre en évidence une différence morphologique ou anatomique entre les deux formes ont échoué. Des infestations expérimentales croisées à partir d'individus provenant des arcs I et IV devraient permettre de confirmer ou d'infirmer l'hypothèse de l'hétérogénéité de la population de Microcotyle chrysophrii.

\section{Bibliographie}

Ancona V.D. (1950) : Détermination et différenciation du sexe chez les Poissons. Ed. Coll. Intern. C.N.R.S., 1951, 31, 92-112.

Audouin J. (1962): La Daurade de l'Etang de Thau. Rev. Trav. Inst. Pêches Manti., 26, 106-126.

Euzet L., Noisy D.: Microcotyle chrysophrii Van Beneden et Hesse, 1863 (Monogenea, Microcotylidae), parasite du Téléostéen Sparus aurata: précisions morpho-anatomiques sur l'adulte et l'oncomiracidium (sous presse).

Lambert A., Maillard C. (1975): Répartition branchiale de deux Monogènes: Diplectanum aequans (Wagener, 1857) Diesing, 1858 et D. laubieri Lambert et Maillard, 1974 (Monogenea, Monopishocotyliea) parasites simultanés de Dicentrarchus labrax (Téléostéen). Ann. Parasitol. Hur Comp., 50, 691-699.

Lasserre G. (1974) : Recherche sur la dynamique des populations de Daurades royales Sparus aurata L., des régions de Sète et d'Arcachon. Thèse Lab. Hydrobiologie marine, Montpellier, 214 p.

Lewellyn J. (1962): The life histories and population dynamics of Monogenean gill parasites of Trachurus trachurus (L.). J. Mar. Biol. Ass. U.K., 42, 587-600.

Van Beneden P.-J., Hesse C.-E. (1863) : Recherches sur les Bdellodes ou Hirudinées et les Trématodes marins. Mem. Acad. Roys. Sci. Belgique, 168 p. 